\title{
Use of kilovoltage $X$-ray volume imaging in patient dose calculation for head-and-neck and partial brain radiation therapy
}

\author{
Weigang Hu'1, Jinsong Ye2, Jiazhou Wang ${ }^{1}$, Xuejun Ma1 and Zhen Zhang**1
}

\begin{abstract}
Background: To evaluate the accuracy of using kilovoltage $x$-ray cone-beam computed tomography (kV-CBCT) imaging for in vivo dose calculations.

Methods: A Region-of-Interest (ROI) CT number mapping method was developed to generate the cone-beam CT number vs. relative electron density calibration curve for 3D dose calculations. The stability of the results was validated for three consecutive months. The method was evaluated on three brain tumors and three head-and-neck tumor cases. For each patient, kV-CBCT images were acquired on the first treatment day and two-week intervals on the Elekta $\mathrm{XVI}$ system. The delivered dose distributions were calculated by applying the patients' treatment plans to the kV-CBCT images. The resulting dose distributions and dose volume histograms (DVHs) of the tumor and critical structures were compared to the original treatment plan.

Results: The kV-CBCT electron density calibration was stable within 1.5\% over a three-month period. The DVH and dose distribution comparison based on the planning CT and the initial kV-CBCT showed good agreements for majority of cases. The doses calculated from the planning CT and kV-CBCT were compared on planes perpendicular to the beam axes and passing through the isocenter. Using $\gamma$ analysis with a criterion of $2 \mathrm{~mm} / 2 \%$ and a threshold of $10 \%$, more than $99.5 \%$ of the points on the iso-planes exhibited $\gamma<1$. For one patient, $\mathrm{kV}-\mathrm{CBCT}$ images detected $5.8 \%$ dose variation in the right parotid due to tumor shrinkage and patient weight loss.

Conclusions: $\mathrm{ROI}$ mapping method is an effective method for the creation of kV-CBCT electron density calibration curves for head-and-neck and brain tumor patients. Dose variations as monitored using kV-CBCT imaging suggest that some patients can benefit from adaptive treatment plan re-optimization.
\end{abstract}

\section{Background}

Patients with head-and-neck and definitive brain tumor are routinely treated with intensity-modulated radiotherapy (IMRT) to enable delivery of highly conformal dose distribution to the tumor while sparing surrounding critical structures. Precise target localization is important for such treatments [1-3]. Ideally, the cumulative dose delivered over the whole treatment course should match the total planned dose. However, many uncertainties can be incurred due to patient set-up, anatomic changes and the organ motions during the course of treatment. Barker JL Jr. et al. reported that relative median loss in gross tumor

* Correspondence: zhenzhang6@yahoo.com

1 Department of Radiation Oncology, Cancer Hospital, Department of Oncology, Shanghai Medical college, Fudan University, Shanghai, China Full list of author information is available at the end of the article volume was $69.5 \%$ and measurable anatomic changes were found throughout the fractionated radiotherapy in head-and-neck patients[4]. As a result of these changes the actual delivered dose deviates from the original planned dose distribution, potentially affecting the tumor control and the normal tissue complication rates.

Cone-beam computed tomography (CBCT) systems mounted on the linear accelerator has become available for image-guided radiotherapy (IGRT). Currently, there are two types of commercially available $\mathrm{CBCT}$ imaging systems: (1) the $\mathrm{kV}-\mathrm{CBCT}$ system, which includes the Varian On-Board-Imaging (OBI) (Varian Medical Systems, Palo Alto, CA) and the Elekta XVI Synergy system (Elekta, Stockholm, Sweden); and (2) the Siemens MVision system (Siemens Medical Solutions, Malvern, PA) 
[5-7]. In our hospital, we commissioned an Elekta Synergy $^{\text {twm }}$ accelerator with on-board $\mathrm{kV}-\mathrm{CBCT}$ in 2006. The main clinical application of $\mathrm{CBCT}$ is to improve the geometric accuracy of target localization in radiation therapy, where the volumetric images of patient acquired immediately before the treatment are registered to the reference planning CT images to correct the patient setup error $[8,9]$. KV-CBCT imaging has shown enough soft tissue contrast and spatial resolution for soft-tissue based setup, but the image quality is affected by the acquisition parameters. In principle the $\mathrm{kV}-\mathrm{CBCT}$ data set can be used to calculate the dose distribution, which means that the planned dose distribution can be evaluated and verified on every treatment day [10]. In order to use CBCT images for dose calculation, the image pixel values need to be converted from dimensionless CT numbers to either electron or physical density. Methods for calibrating conventional fan-beam CT to electron density have been widely used in clinical dose calculation[11]. However, compared to conventional CT scanners, $\mathrm{kV}$-CBCT images have increased artifacts and reduced contrast due to photon scatter. As a result, the calibration of $\mathrm{kV}$-CBCT images for dose calculation is an active area of research [12-15].

The purpose of this study is to assess the feasibility of using a mapping method to calibrate the $\mathrm{kV}$-CBCT images for dose calculation in head-and-neck and definitive brain tumor radiation treatments. By monitoring the dose that patient receives from each fraction, physicians will be able to track the dose distribution during the course of radiation therapy and modify the treatment plan as needed based on the actual dose delivered.

\section{Methods}

\section{KV CBCT data acquisition}

The $\mathrm{kV} \mathrm{CBCT}$ images were acquired on a linear accelerator equipped with an integrated $\mathrm{kV} \mathrm{X}$-ray volumetric imaging system (Elekta, Synergy S, XVI, Crawley, UK). For imaging the head-and-neck and brain tumor patients, we used the following parameters: $100 \mathrm{kVp}$, S20 collimator and F0 filter, total $65 \mathrm{mAs}$ and a high-resolution reconstruction $(512 \times 512)$. A total of about 650 projections were acquired in a full rotation. The CBCT images were reconstructed with slice thickness of $2.5 \mathrm{~mm}$ and then transferred to the treatment planning system (TPS, Philips Pinnacle3 V8.0d, Fitchburg, WI, USA) for image registration and dose calculations.

\section{KV-CBCT Stability}

A phantom, Catphan-600 module CTP503 (Phantom Laboratory, NY) was used to evaluate the stability and uniformity of the $\mathrm{CBCT}$ numbers. The phantom has seven embedded rods made of different materials: air, PMP, LDPE, polystyrene, acrylic, Delrin and Teflon. Their electron densities relative to water range from 0.00 to 2.16. The CBCT image of the phantom was acquired every month for three consecutive months, and the CBCT numbers were obtained from the TPS and the relative electron densities were recorded accordingly. We also evaluated the maximal fluctuation in CBCT numbers on the image uniformity module part of the phantom.

\section{Calibration of relative electron density}

For the dose calculation in a treatment planning system, the relative electron density or physical density of each voxel of the $\mathrm{CT}$ images is required for inhomogeneity corrections [11]. In this study, calibration of conventional CT (AcQsim CT Simulator, Philips Medical System, Cleveland, $\mathrm{OH}$ ) number to physical density was performed on a CT phantom (CIRS model 062, Norfolk, VA). However, each individual patient's CBCT scan has a different scatter component that affects the HU measured in the image. A significant dose error was observed if we directly applied the calibration method for conventional CT to $\mathrm{kV}$-CBCT.

We used a Region-of-interest (ROI) CT number mapping method similar to Richter's report to generate the CT number to physical density conversion curve for the dose calculation [14]. This process was applied to a single patient with head and neck cancer. The CBCT images for this patient were acquired at the same day of planning $\mathrm{CT}$, so that there was no visible change in patient anatomy between the two images. A brief description of the calibration progress is as follows: (1) register the planning $\mathrm{CT}$ images and $\mathrm{kV}-\mathrm{CBCT}$ images in the ADAC Pinnacle treatment planning system; (2) map the regions of interests (ROIs) from conventional CT dataset to the CBCT dataset, and record the mean $\mathrm{CBCT}$ number values of these ROIs, and (3) Generate the kV-CBCT numbers to physical density calibration curve based on the density values measured on the conventional $\mathrm{CT}$.

\section{Clinical Implementation}

Three head-and-neck cases and three brain patients with different tumor sites treated on Elekta Synergy were selected for retrospective evaluation of the accuracy of CBCT-based dose calculations. The head-and-neck cases included two natural killer/T-cell (NK/T) lymphoma cases and one nasopharyngeal carcinoma (NPC) case. For all patients, conventional CT was acquired with slice thickness of $5 \mathrm{~mm}$ and the target and critical structures were delineated by the attending physicians. IMRT plans were designed according to the physician's prescriptions with beams of $6 \mathrm{MV}$. The beam angles were $0,50,110$, 250 and 310 degree for NK/T cases, and 0, 45, 90, 120, 160, 200, 240, 280 and 320 degree for NPC cases. For brain cases, gantry angles were $0,60,230,300$ and 45 
degree with a 90-degree couch kick. Two sets of CBCT images were acquired, one on the first treatment day and the other on two weeks later. The patients were initially set up to the skin markers then followed with a CBCT scanning. The CBCT images were acquired according to the appropriate protocol and then reconstructed with slice thickness of $2.5 \mathrm{~mm}$. All images were transferred to treatment planning system for analysis. For each case, the $\mathrm{CBCT}$ images were first registered to the conventional $\mathrm{kV}-\mathrm{CT}$ images using an automatic registration method based on normalized mutual information algorithm, and then manual adjustments were performed to achieve the optimal match. A second set of CBCT images were acquired two weeks later and registered to the reference planning CT with the same method. The contours were mapped from $\mathrm{CT}$ to $\mathrm{CBCT}$ images with slight changes if them were beyond the skin.

For dose calculation, all the parameters (e.g., iso-center location, beam angles, MLC shapes, and monitor units) in the initial treatment plan were applied to the $\mathrm{kV}-\mathrm{CBCT}$ images, and then the dose distribution was recalculated based on the new calibration curve. The dose calculation was performed in the Pinnacle treatment planning system using the collapsed cone superposition convolution algorithm with an isotropic $2 \mathrm{~mm}$ dose grid resolution. The contours delineated on the conventional CT were also mapped onto the kV-CBCT image data sets. Finally, the initial dose distribution matrix calculated on the planning CT was imported in the treatment planning system and displayed on the $\mathrm{kV}-\mathrm{CBCT}$ dataset using scripts developed in-house. Dose volume histogram and the dose to tumor and normal structures were compared on the two image data sets. The differences in the dose distributions of the two plans were analyzed using $\gamma$ analysis along planes through the isocenter perpendicular to each beam axis using commercial software (MapCheck, Version4.0, Sun Nuclear, Melbourne, FL)[16].

\section{Results}

\section{The stability of $\mathrm{kV}$-CBCT numbers}

Because most patients complete their treatment courses within five weeks, we consider the three-month length of the stability test to be adequate. The maximal difference in $\mathrm{CBCT}$ numbers was 21 , with a maximum standard error of less than $1.5 \%$. The stability of $\mathrm{kV}-\mathrm{CBCT}$ number and electron density indicates acceptable overall performance of the $\mathrm{kV}-\mathrm{CBCT}$ system. The $\mathrm{kV}-\mathrm{CBCT}$ images of the uniformity section of the phantom shows the maximal fluctuation of the CBCT numbers is \pm 35 Hounsfield unit $(\mathrm{HU})$, which translates to a fluctuation of approximately $1 \%$ in electron density values.

\section{Conversion of $\mathrm{kV}-\mathrm{CBCT}$ numbers to relative electron density}

A total of 13 different ROIs were used in generating the conversion curve, which include air, skin, muscle, brain stem, spinal cord, parotid gland, outer bone, inner bone, tooth and other structures. Table 1 shows the CBCT numbers and their corresponding physical density values. The calibration curves, as shown in Figure 1, were implemented in treatment planning system for the dose calculations. Large discrepancies were noted from these two curves. In particular, some discontinuous steps were observed on the calibration curve of $\mathrm{kV}-\mathrm{CBCT}$ images.

\section{Clinical cases}

Only minimal changes and deformations were observed in the anatomical structures on the patients' first CBCT images as compared with the reference planning CT. The DVHs of one NK/T lymphoma case (patient1), one NPC case (patient2) and one brain tumor case (patient3) are shown in Figure 2 as an example. The solid lines represented the DVHs based on conventional CT images and the dash lines were based on the dose calculated from the KV-CBCT images. Figure 3(a)-(c) are the dose distributions on the transverse planes of the three patients. The left images represent dose distributions based on the $\mathrm{kV}$ $\mathrm{CBCT}$ and the right had images represent the dose on $\mathrm{kV}-\mathrm{CT}$ images. There is no significant dose difference between the conventional $\mathrm{CT}$ images and $\mathrm{kV}-\mathrm{CBCT}$ images.

For all clinical cases, the dose comparison was performed at a plane through the isocenter for each individual beam. Good agreement was found between the conventional CT and the first $\mathrm{kV}$-CBCT based dose calculations. Using the $\gamma$ analysis with a criterion of $2 \mathrm{~mm}$ and $2 \%$ and a threshold of $10 \%$, more than $99.5 \%$ of the points at the iso-plane have the $\gamma$ value less than 1.0. Table 2 shows the distance to agreement (DTA) and gamma analysis results of the three cases. For most of the

Table 1: The densities and CBCT numbers.

\begin{tabular}{|c|c|c|c|c|c|c|c|c|c|c|c|c|c|}
\hline Region of interest (ROI) & 1 & 2 & 3 & 4 & 5 & 6 & 7 & 8 & 9 & 10 & 11 & 12 & 13 \\
\hline CBCT Numbers (HU) & 0 & 1379 & 1500 & 1950 & 1990 & 2000 & 2103 & 2158 & 2468 & 2500 & 2670 & 3293 & 3847 \\
\hline $\begin{array}{l}\text { Density } \\
\left(\mathrm{g} / \mathrm{cm}^{3}\right)\end{array}$ & 0.0 & 0.0 & 0.9 & 0.9 & 1.02 & 1.03 & 1.06 & 1.09 & 1.30 & 1.50 & 1.62 & 1.84 & 1.86 \\
\hline
\end{tabular}

The densities and $\mathrm{CBCT}$ numbers for generating the $\mathrm{CBCT}$ calibration curve 


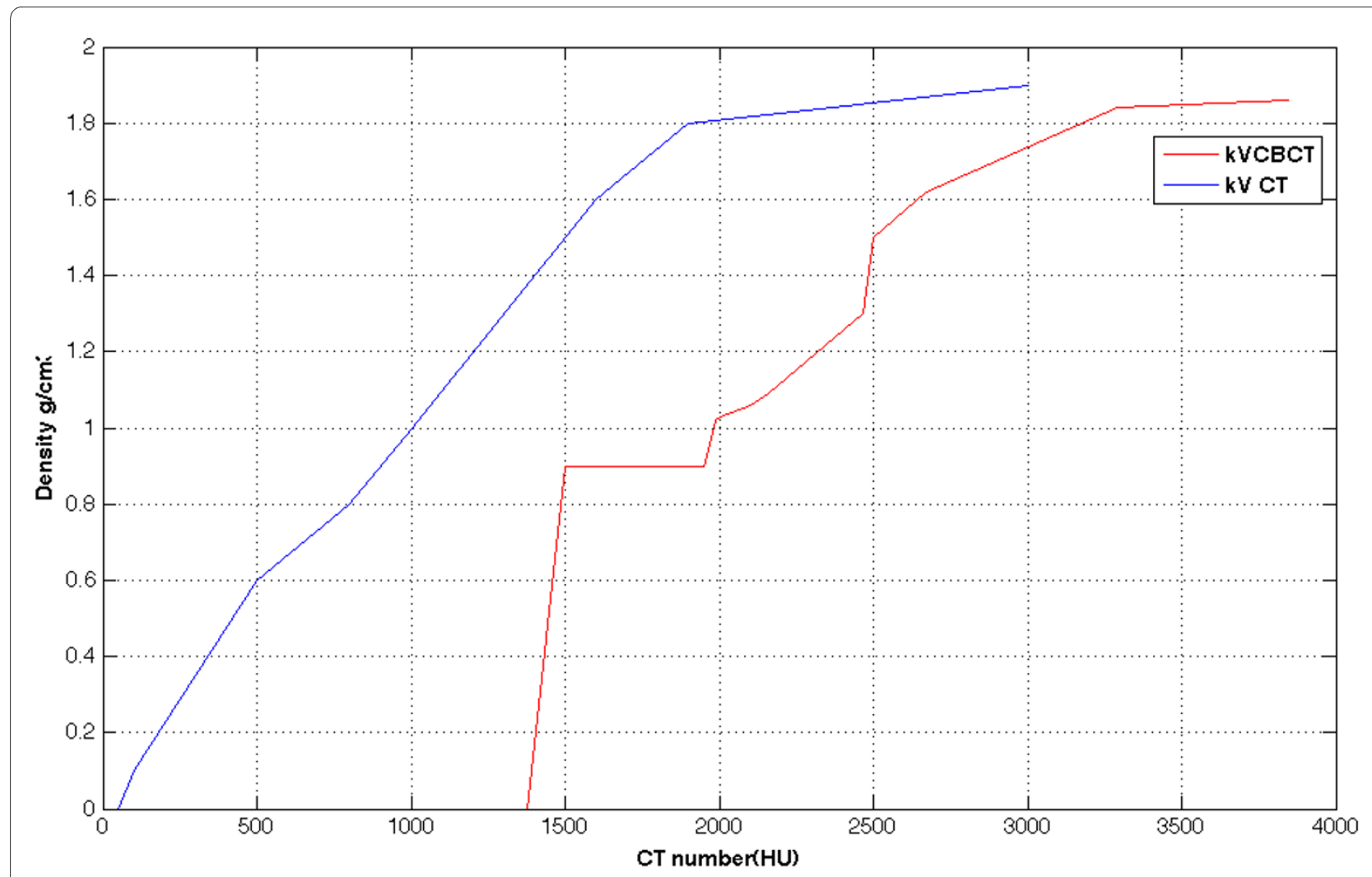

Figure 1 Calibration curves for kV CT and kV CBCT. The calibration curves for kV-CT and kV-CBCT based dose calculations in the treatment planning system.

beams, the pass rate for distance to agreement were better than $96 \%$ except one beam which has the data of $94.5 \%$.

Table 3 shows the dose to the tumor and some normal structures of the three patients in the planning CT data sets and the first CBCT data sets. The differences of the dose to tumor and some normal tissues were within $1 \%$ and $3.2 \%$, respectively. The difference of maximal dose in tumor is $0.49 \%$ and in normal structures are $3.15 \%$.

Five out of six patients didn't show significant anatomy changes and setup variations between the first CBCT images and the second CBCT images. But for one NK/T patient (patient5), a slight anatomical change in the patient's skin contour and air cavity was found in the second $\mathrm{CBCT}$ images compared to the conventional CT images, as shown in Figure 4. The dose comparisons of the reference $\mathrm{kVCT}$, the 1 st and 2 nd $\mathrm{kV}$ CBCTs for that patient are listed in Table 4. On the first treatment day, the dose difference in gross tumor volume (GTV), clinical tumor volume (CTV) and planning tumor volume (PTV) between reference CT and cone beam CT (kV-CBCT1) were $0.98 \%, 0.54 \%, 0.54 \%$, respectively. The maximal dose difference was found on the spinal cord (-1.87\%). For the second cone beam CT (kV-CBCT2) acquired two weeks later, the maximal dose difference of spinal cord increased to $3.77 \%$, and the maximal dose difference was found in the right parotid (5.81\%). While for tumor and other structures, the dose agreement was still within $1.0 \%$.

\section{Discussion}

On-board CBCT volumetric imaging can improve the accuracy of radiation therapy in two aspects, namely target localization and delivered dose verification $[12,17,18]$. By acquiring 3D CBCT images with patient on the treatment couch just before the treatment delivery, patient setup error can be corrected and the accuracy of target positioning localization accuracy can be improved. CBCT image data sets obtained throughout the treatment course can be used for dose calculation, hence providing a clinical quality assurance tool for radiotherapy. However, the CBCT image quality is susceptible to many factors, such as scattering, beam hardening effects and organ motion, etc [19]. Morin et al studied dose calculation based on MV CBCT images and reported that the MV CBCT could be used to estimate the dose variation due to the anatomical changes in the head-and-neck region [20]. In this study, we investigated the feasibility and the accuracy of using $\mathrm{kV} \mathrm{CBCT} \mathrm{images} \mathrm{for} \mathrm{direct}$ 

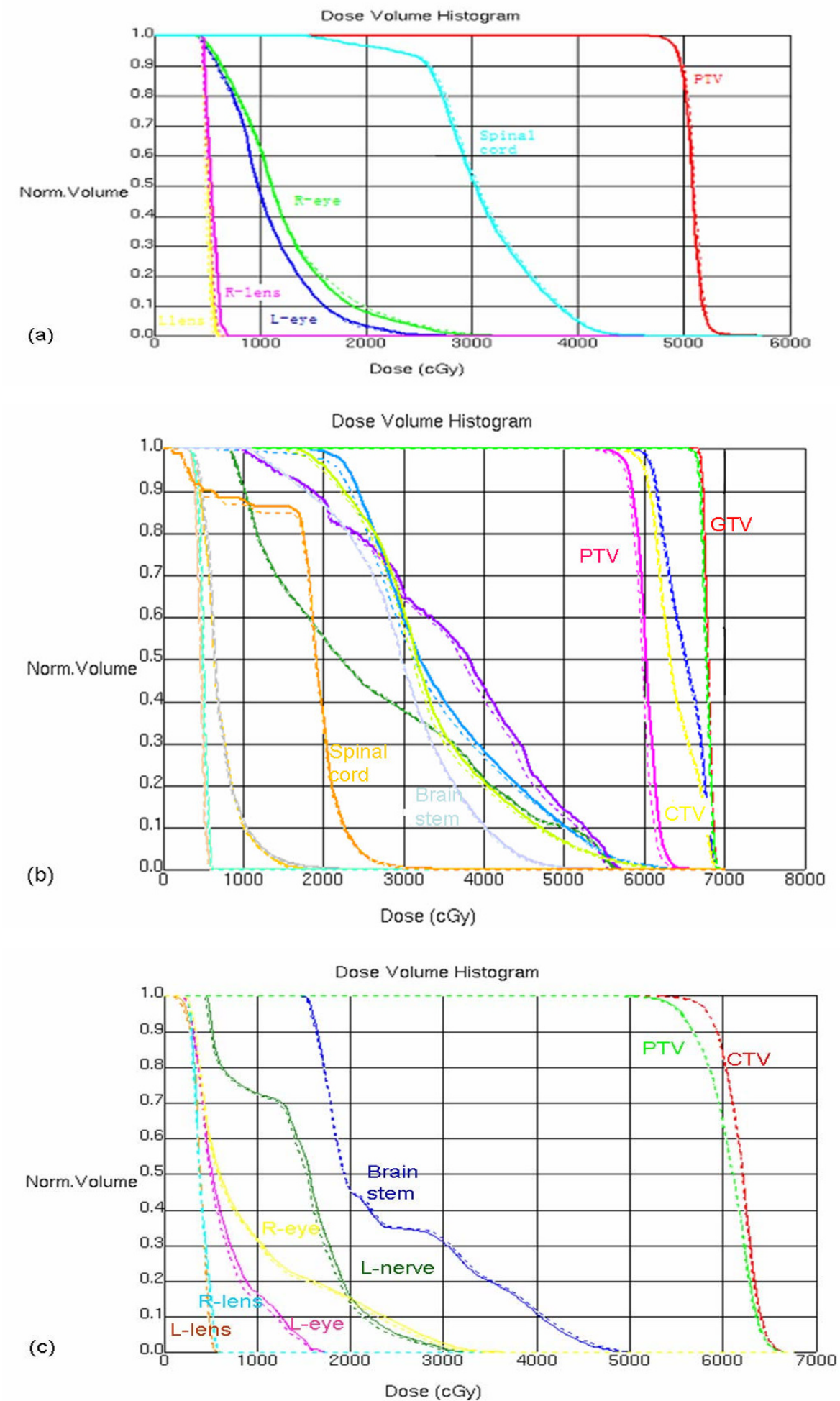

Figure 2 DVH displays of three clinical cases. The DVHs of three cases: one NK/T lymphoma (a), one NPC (b) and one Brain (c). The solid lines represent the dose based on conventional $C T$ and the dash lines represent the dose based on $\mathrm{kV} \mathrm{CBCT}$. 


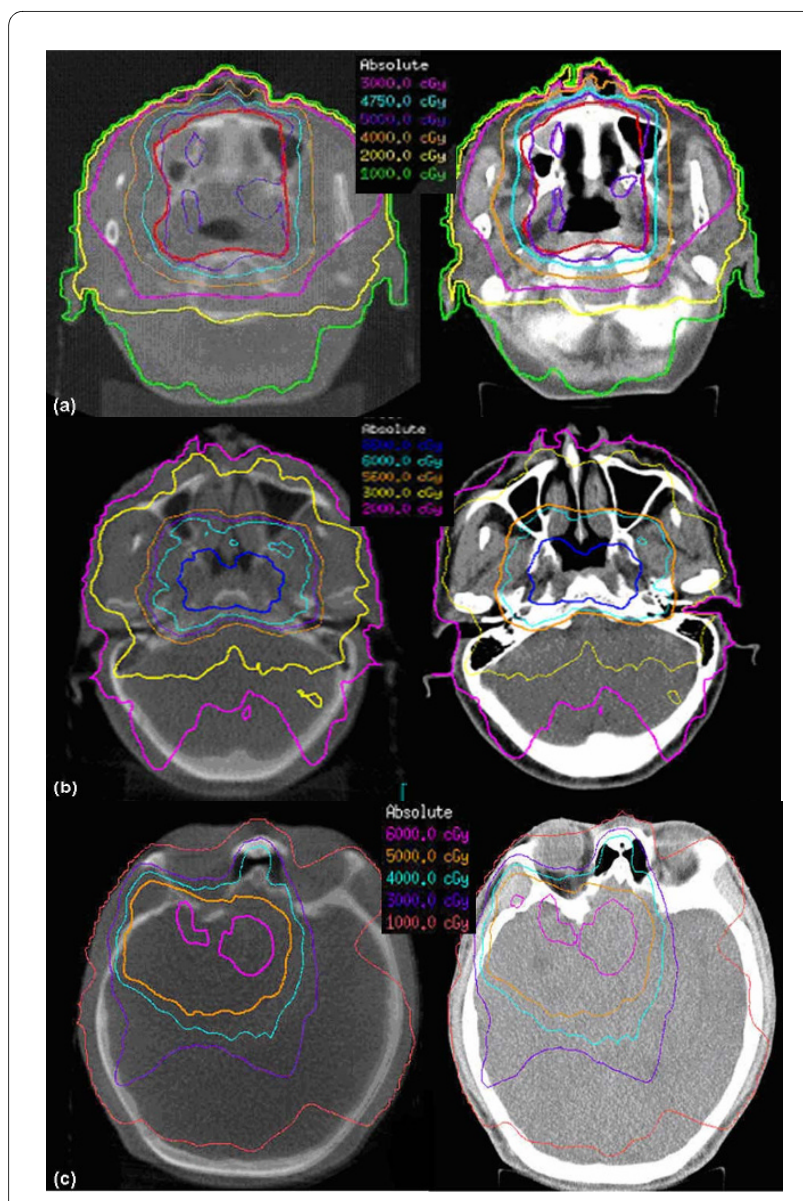

Figure 3 Dose distributions of three clinical cases. The transverse views of dose distributions of the NKT lymphoma (a), NPC (b) and brain tumor (c). Left: calculated dose based on CBCT images; Right: calculated dose based on planning CT images. They show good agreement on both relative high and low isodoses.

dose calculation in head-and-neck and brain tumor radiotherapy with a simple and effective method.

The relative electron density can directly affect the dose calculation accuracy when inhomogeneity correction is involved. Unlike conventional $\mathrm{kV}$ CT or MV CBCT, kV$\mathrm{CBCT}$ has a larger scatter radiation component and the image quality suffers from the beam hardening effect [2123]. It has been reported that the effect of scatter radiation can be partly corrected or reduced by calibrating the $\mathrm{kV}-\mathrm{CBCT}$ system $[24,25]$. The conventional CT number to the relative electron density conversion was performed with a CT number calibration phantom embedded with different types of tissue-equivalent inserts. However if such method was used directly for $\mathrm{kV}$-CBCT, a dose calculation error can be introduced. Based on our tests, if we used the calibration curve generated by the phantom directly, the dose difference between first CBCT and planning $\mathrm{CT}$ would be more than $5 \%$, which agrees with the reports from Yang et al and Tucking et al [12,26].
In this study, we used the ROI mapping method to generate calibration curve for $\mathrm{kV}$ CBCT image-based dose calculation [14]. Obviously, accurate image registration is needed for this method. The registration of different image modalities is widely used in radiotherapy for delineating the region of interests[27]. As the registration algorithms in the commercial treatment planning systems generally use rigid body transformations, we selected the head-and-neck and brain tumor cases for our study, where this assumption was generally valid.

The calibration curve for $\mathrm{kV}-\mathrm{CBCT}$ is different from that for conventional CT. The conventional CT number is zero for the air outside the patient skin in the planning system; however, the CBCT number in such situation is much greater than zero. The mean $\mathrm{CBCT}$ value in the air around the skin is 1379 for the selected case, similar to other report [14]. The steps in the $\mathrm{kV} \mathrm{CBCT}$ electron density conversion curve is mainly caused by scatter and beam hardening effects.

A good agreement of the calculated doses to the tumor and normal structures was found between the conventional $\mathrm{CT}$ and the first $\mathrm{kV}-\mathrm{CBCT}$ images because there were virtually no anatomical changes between these images. The maximal dose deviation was found in the eye mainly due to the residual registration error and contour deviations, as the slice thickness was $5 \mathrm{~mm}$ for the conventional $\mathrm{CT}$ and $2.5 \mathrm{~mm}$ for $\mathrm{CBCT}$ images. The structures near the skin showed larger differences. The DTA and $\gamma$ index analysis results also showed the good agreement between $\mathrm{kV}-\mathrm{CBCT}$ based and conventional $\mathrm{CT}$ based dose calculation. Richter et al. used the same method and reported the dose difference between the planning CT and CBCT was $1.36 \% \pm 1.96 \%$ in head patients with three-dimensional conformal plans. Our data showed the difference was within $1 \%$ of the target, which was consistent with their result. Our results demonstrated that the mapping method for $\mathrm{CBCT}$ correction is accurate both for three-dimensional conformal plans and IMRT plans in head and brain cases.

Furthermore, we generated the density conversion table based on one patient and applied the same table to the other patients. There were only a small discrepancy between the doses calculated by using $\mathrm{kV} \mathrm{CBCT}$ and conventional $C T$ in all 6 cases with different tumor locations. This result suggests that, for head-and-neck and brain patients, variations in the scatter effect in imaging different tumor sites is relatively small from patient to patient, and therefore it is reasonable to use the same electron density conversion curve for $\mathrm{kV}$ CBCT based dose calculation. Compared to the patient group based conversion table in report of Richter et al. or CT-based HU mapping method in Mathilda et al., this specific case mapping method is less complex to develop and implement, but it is limited to the preset scanning parameters. 
Table 2: The comparison of iso-plane dose distributions.

\begin{tabular}{|c|c|c|c|}
\hline Patients & Beam index & $\begin{array}{c}\text { DTA } \\
\text { (3 mm, 3\%, 10\%) } \\
\text { Pass rate (\%) }\end{array}$ & $\begin{array}{c}\text { Gamma index analysis } \\
(2 \mathrm{~mm}, 2 \%, 10 \%, \gamma<1) \\
\text { Pass rate }(\%)\end{array}$ \\
\hline \multirow[t]{5}{*}{$\mathrm{NK} / \mathrm{T}$} & 1 & 99.0 & 100.0 \\
\hline & 2 & 100.0 & 100.0 \\
\hline & 3 & 99.4 & 100.0 \\
\hline & 4 & 99.5 & 100.0 \\
\hline & 5 & 99.2 & 100.0 \\
\hline \multirow[t]{9}{*}{ NPC } & 1 & 97.2 & 99.5 \\
\hline & 2 & 98.8 & 99.8 \\
\hline & 3 & 99.8 & 99.8 \\
\hline & 4 & 98.9 & 100.0 \\
\hline & 5 & 96.3 & 99.6 \\
\hline & 6 & 94.5 & 99.9 \\
\hline & 7 & 99.8 & 100.0 \\
\hline & 8 & 99.5 & 100.0 \\
\hline & 9 & 99.0 & 100.0 \\
\hline \multirow[t]{5}{*}{ Brain Tumor } & 1 & 99.7 & 100.0 \\
\hline & 2 & 99.8 & 100.0 \\
\hline & 3 & 96.3 & 100.0 \\
\hline & 4 & 100.0 & 100.0 \\
\hline & 5 & 100.0 & 100.0 \\
\hline
\end{tabular}

The comparison of iso-plane dose distributions based on conventional CT and KVCBCT for 3 clinical cases using distance to agreement (DTA) and gamma index analysis in all the beams.

Overall, our study showed good accuracy in CBCT based dose calculation. However, it is not recommended to replace the conventional planning CT by $\mathrm{kV} \mathrm{CBCT}$ for the purpose of treatment planning as the inferior image quality of $\mathrm{kV} \mathrm{CBCT}$ may affect the accuracy of target and normal structures delineation.

The $\mathrm{kV} \mathrm{CBCT} \mathrm{can} \mathrm{also} \mathrm{be} \mathrm{used} \mathrm{to} \mathrm{evaluate} \mathrm{the} \mathrm{dose} \mathrm{to}$ tumor or the normal structures. In this study, one NK/T patient had slight changes in anatomy after two weeks' treatment, dose variations were found in the spinal cord and the right parotid gland. These results suggested that even within a relatively short period such as 2 weeks, dose verification based on CBCT or CT will be necessary for certain patients to account for dosimetric effects due to patient anatomical changes.

Anatomic changes for head-and-neck patients, including nodal mass shrinkage and patient weight loss during the course of radiation therapy, can occur $[28,29]$. For these cases, repeat $\mathrm{CT}$ imaging and re-planning may be essential to ensure the adequate dose delivered to the tumor and proper sparing of the surrounding sensitive structures.
Technically, the $26 \mathrm{~cm}$ field of view for the S20 collimator may limit the use of $\mathrm{kV} \mathrm{CBCT}$ for dose calculation of patients with beams going through their shoulders. However for most head-and-neck patients, the FOV is sufficiently large to evaluate the dose to PTV, brain stem, spinal cord, eyes and parotid glands. For those patients who receive thoracic or pelvic treatment, S20 is not large enough to encompass all the structures and skin. Dose verification for other sites is part of our future research.

\section{Conclusions}

ROI mapping method is a feasible method to overcome the effects of scatter for generating the $\mathrm{kV} \mathrm{CBCT}$ relative electron density calibration curve for head-and-neck cancer and brain tumor patients. Dose variations as monitored using $\mathrm{kV}$ CBCT imaging were observed in a relatively short period of two weeks, which suggests potential benefits of adaptive treatment plan re-optimization for certain head-and-neck and brain tumor patients.

\section{Declaration of competing interests}

The authors declare that they have no competing interests. 
Table 3: Dose comparisons in targets and normal tissues.

\begin{tabular}{|c|c|c|c|c|}
\hline \multicolumn{2}{|c|}{ Patients } & \multirow{2}{*}{$\begin{array}{r}\text { 1st kVCBCT (Gy) } \\
51.03\end{array}$} & \multirow{2}{*}{$\begin{array}{c}\text { kVCT (Gy) } \\
51.28\end{array}$} & \multirow{2}{*}{$\begin{array}{c}\text { Difference (\%) } \\
0.49 \%\end{array}$} \\
\hline NK-T lymphoma & 1PTV: mean dose & & & \\
\hline & 1Right-eye: mean dose & 11.68 & 11.76 & $0.70 \%$ \\
\hline & 1Left-eye: mean dose & 10.54 & 10.58 & $0.37 \%$ \\
\hline & 1Spinal cord: max dose & 47.14 & 47.65 & $1.07 \%$ \\
\hline & 1Whole body: max dose & 59.18 & 59.44 & $-0.43 \%$ \\
\hline \multirow{8}{*}{$\begin{array}{c}\text { NPC } \\
\text { (patient2) }\end{array}$} & 2GTV: mean dose & 67.8 & 67.78 & $-0.03 \%$ \\
\hline & 2CTV: mean dose & 64.9 & 64.89 & $-0.02 \%$ \\
\hline & 2PTV: mean dose & 63.76 & 63.78 & $0.03 \%$ \\
\hline & 2Brain Stem: max dose & 53.83 & 54.36 & $0.98 \%$ \\
\hline & 2Spinal cord: max dose & 32.85 & 32.73 & $-0.34 \%$ \\
\hline & 2L-parotid: mean dose & 34.92 & 34.11 & $-2.37 \%$ \\
\hline & 2R-parotid: mean dose & 33.38 & 32.91 & $-1.41 \%$ \\
\hline & 2Whole body: max dose & 70.73 & 70.12 & $0.87 \%$ \\
\hline \multirow{6}{*}{$\begin{array}{l}\text { Brain Tumor } \\
\text { (patient3) }\end{array}$} & 3CTV: mean dose & 61.85 & 61.84 & $-0.02 \%$ \\
\hline & 3PTV: mean dose & 60.64 & 60.57 & $-0.11 \%$ \\
\hline & 3Brain: max dose & 50.13 & 50.02 & $-0.22 \%$ \\
\hline & 3Left-eye: mean dose & 19.42 & 19.4 & $-0.13 \%$ \\
\hline & 3Right-eye: mean dose & 9.94 & 9.64 & $-3.15 \%$ \\
\hline & 3Whole body: max dose & 67.21 & 67.66 & $0.66 \%$ \\
\hline
\end{tabular}

Dose comparisons of the first kVCBCT to the planning kVCT in targets and normal tissues for the three clinical cases (NK-T lymphoma, NPC and Brain Tumor). The difference of maximal dose in tumor is $0.49 \%$ and in normal structures are $-3.15 \%$. The numbers before the contours are the indexes of patients, 1PTV means the PTV in patient1.
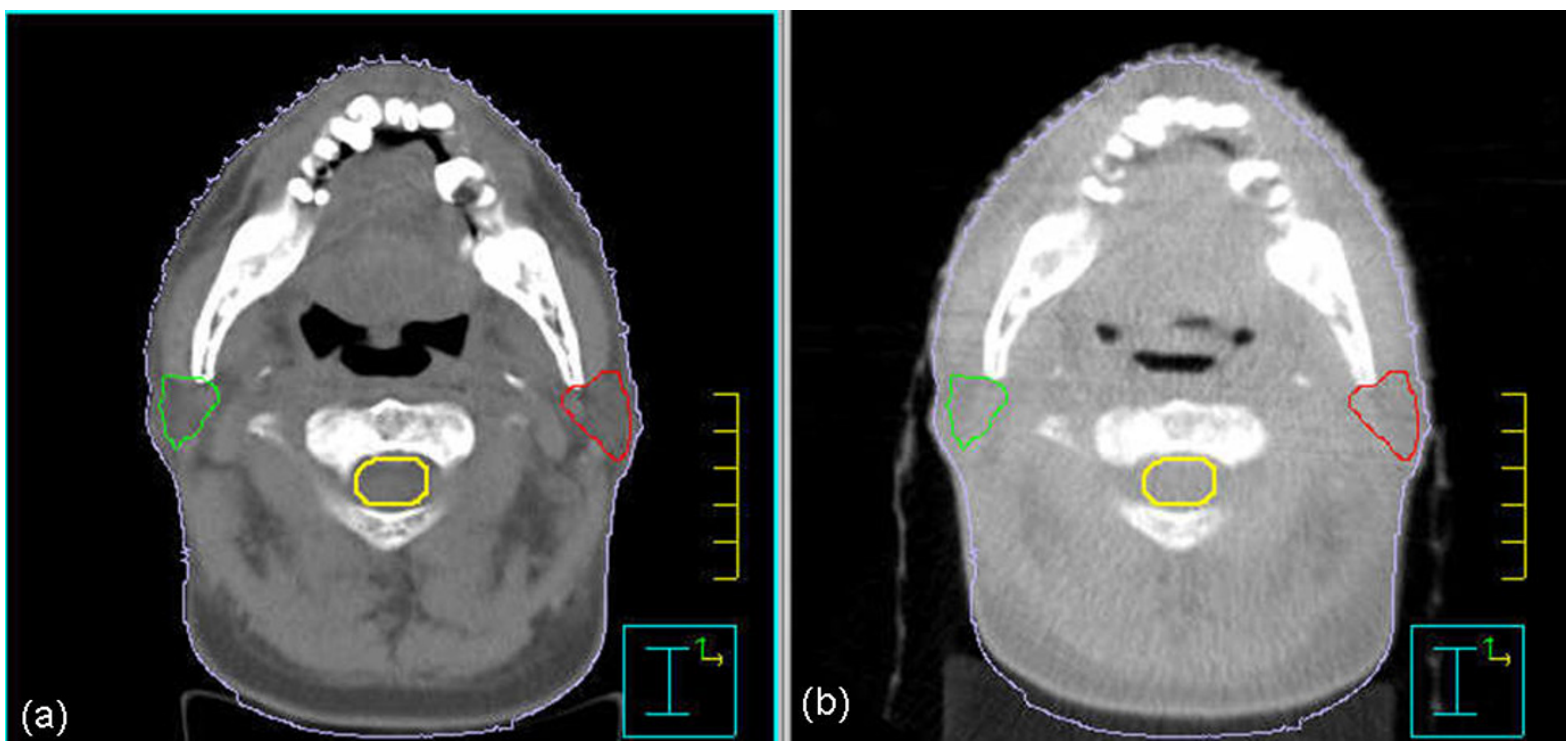

Figure 4 The transverse views of $C T$ and the 2 nd CBCT. The transverse views of the reference $C T$ (a) and cone beam $C T$ (b) after two weeks of the treatment. A slight change happened in the external contour and air cavity. 
Table 4: Dose comparisons of the first and second CBCT images.

\begin{tabular}{|c|c|c|c|c|c|c|c|c|}
\hline & \multicolumn{5}{|c|}{ Mean dose (Gy) } & \multicolumn{3}{|c|}{ Maximal dose (Gy) } \\
\hline & GTV & CTV & PTV & Left-parotid & Right-parotid & Brain-stem & Spinal-cord & Whole-body \\
\hline $\mathrm{kV} C \mathrm{~T}$ & 50.72 & 51.04 & 51.02 & 32.14 & 30.40 & 50.92 & 31.76 & 55.78 \\
\hline $1^{\text {st }} \mathrm{kV}$ CBCT & 50.23 & 50.77 & 50.74 & 31.88 & 30.57 & 51.21 & 31.17 & 55.63 \\
\hline $\begin{array}{l}\text { Dose difference between } 1^{\text {st }} \mathrm{kV} \\
\text { CBCT and } \mathrm{kV} \mathrm{CT( \% )}\end{array}$ & $0.98 \%$ & $0.54 \%$ & $0.54 \%$ & $0.8 \%$ & $-0.56 \%$ & $0.57 \%$ & $-1.87 \%$ & $-0.26 \%$ \\
\hline $2^{\text {nd }} k V$ CBCT & 50.37 & 50.77 & 50.76 & 32.56 & 28.73 & 51.36 & 32.96 & 55.71 \\
\hline $\begin{array}{l}\text { Dose difference between } 2^{\text {nd }} \mathrm{kV} \\
\text { CBCT and } \mathrm{kV} \mathrm{CT( \% )}\end{array}$ & $0.7 \%$ & $0.53 \%$ & $0.52 \%$ & $-1.3 \%$ & $5.81 \%$ & $0.86 \%$ & $3.77 \%$ & $-0.11 \%$ \\
\hline
\end{tabular}

\section{Authors' contributions}

Each author has participated sufficiently in the work to take public responsibility for appropriate portions of the content. JY, ZZ designed the study. WH, JW performed the study and analysis. XM provided the patients' images. The manuscript was written by $\mathrm{WH}$, all other authors helped and finally approved the final manuscript.

\section{Acknowledgements}

The authors thank the Drs. Lijun Ma and Andrew Huwang for helpful discussions and editing of the paper.

\section{Author Details}

'Department of Radiation Oncology, Cancer Hospital, Department of Oncology, Shanghai Medical college, Fudan University, Shanghai, China and 2Department of Radiation Oncology, Swedish Cancer Institute, Seattle, WA, USA

Received: 18 January 2010 Accepted: 19 April 2010 Published: 19 April 2010

\section{References}

1. Hong TS, Tomé WA, Chappell RJ, Chinnaiyan P, Mehta MP, Harari PM: The impact of daily setup variations on head-and-neck intensitymodulated radiation therapy. Int J Radiat Oncol Biol Phys 2005, 61:779-88.

2. Xing L, Lin Z, Donaldson SS, Le QT, Tate D, Goffinet DR, Wolden S, Ma L, Boyer AL: Dosimetric effects of patient displacement and collimator and gantry angle misalignment on intensity modulataed radiation therapy. Radiother Oncol 2000, 56:97-108.

3. Han C, Chen YJ, Liu A, Schultheiss TE, Wong JY: Actual dose variation of parotid glands and spinal cord for nasopharyngeal cancer patients during radiotherapy. Int J Radiat Oncol Biol Phys 2008, 70:1256-62.

4. Barker JL Jr, Garden AS, Ang KK, O'Daniel JC, Wang H, Court LE, Morrison WH, Rosenthal DI, Chao KS, Tucker SL, Mohan R, Dong L: Quantification of volumetric and geometric changes occurring during fractionated radiotherapy for head-and-neck cancer using an integrated $\mathrm{CT} /$ /inear accelerator system. Int J Radiat Oncol Biol Phys 2004, 59:960-70.

5. Kim GY, Pawlicki T, Le QT, Luxton G: Linac-based on-board imaging feasibility and the dosimetric consequences of head roll in head-andneck IMRT plans. Med Dosim 2008, 33(1):93-9.

6. Oelfke U, Tücking T, Nill S, Seeber A, Hesse B, Huber P, Thilmann C: Linacintegrated kV-cone beam CT: technical features and first applications. Med Dosim 2006, 31:62-70

7. Morin O, Gillis A, Chen J, Aubin M, Bucci MK, Roach M, Pouliot J: Megavoltage cone-beam CT: system description and clinical applications. Med Dosim 2006, 31:51-61.

8. Chang J, Mageras GS, Yorke E, De Arruda F, Sillanpaa J, Rosenzweig KE, Hertanto A, Pham H, Seppi E, Pevsner A, Ling CC, Amols H: Observation of interfractional variations in lung tumor position using respiratory gated and ungated megavoltage cone-beam computed tomography. Int J Radiat Oncol Biol Phys 2007, 67:1548-58.

9. Fatunase T, Wang Z, Yoo S, Hubbs JL, Prosnitz RG, Yin FF, Marks LB: Assessment of the Residual Error in Soft Tissue Setup in Patients Undergoing Partial Breast Irradiation: Results of a Prospective Study Using Cone-Beam Computed Tomography. Int J Radiat Oncol Biol Phys 2008, 70:1025-34

10. Varadhan R, Hui SK, Way S, Nisi K: Assessing prostate, bladder and rectal doses during image guided radiation therapy--need for plan adaptation? J App/Clin Med Phys 2009, 10(3):2883.

11. Thomas SJ: Relative electron density calibration of CT scanners for radiotherapy treatment planning. Br J Radiol 1999, 72:781-6.

12. Yang Y, Schreibmann E, Li T, Wang C, Xing L: Evaluation of on-board kV cone -beam CT (CBCT)-based dose calculation. Phys Med Biol 2007, 52(3):685-705

13. van Zijtveld M, Dirkx M, Heijmen B: Correction of conebeam CT values using a planning CT for derivation of the "dose of the day". Radiother Oncol 2007, 85(2):195-200

14. Richter A, Hu Q, Steglich D, Baier K, Wilbert J, Guckenberger M, Flentje M: Investigation of the usability of conebeam CT data sets for dose calculation. Radiat Oncol 2008, 3:42

15. Guan H, Dong H: Dose calculation accuracy using cone-beam CT (CBCT) for pelvic adaptive radiotherapy. Phys Med Biol 2009, 54(20):6239-50.

16. Low DA, Harms WB, Mutic S, Purdy JA: A technique for the quantitative evaluation of dose distributions. Med Phys 1998, 25:656-61.

17. Thilmann C, Nill S, Tücking T, Höss A, Hesse B, Dietrich L, Bendl R, Rhein B, Häring P, Thieke C, Oelfke U, Debus J, Huber P: Correction of patient positioning errors based on in-line cone beam CTs: clinical implementation and first experiences. Radiat Oncol 2006, 1:16.

18. Grills IS, Hugo G, Kestin LL, Galerani AP, Chao KK, Wloch J, Yan D: ImageGuided Radiotherapy Via Daily Online Cone-Beam CT Substantially Reduces Margin Requirements for Stereotactic Lung Radiotherapy. Int J Radiat Oncol Biol Phys 2008, 70:1045-56.

19. Jaffray DA, Battista JJ, Fenster A, Munro P: X-ray scatter in megavoltage transmission radiography: physical characteristics and influence on image quality. Med Phys 1994, 21:45-60

20. Morin O, Chen J, Aubin M, Gillis A, Aubry JF, Bose S, Chen H, Descovich M, Xia P, Pouliot J: Dose calculation using megavoltage cone-beam CT. Int J Radiat Oncol Biol Phys 2007, 67:1201-10.

21. Ding GX, Duggan DM, Coffey CW: Characteristics of kilovoltage x-ray beams used for cone-beam computed tomography in radiation therapy. Phys Med Biol 2007, 52:1595-615.

22. Siewerdsen JH, Jaffray DA: Cone-beam computed tomography with a flat-panel imager: magnitude and effects of $x$-ray scatter. Med Phys 2001, 28:220-31. 
23. Reitz I, Hesse BM, Nill S, Tücking T, Oelfke U: Enhancement of image quality with a fast iterative scatter and beam hardening correction method for kV CBCT. ZMed Phys 2009, 19(3):158-72.

24. Létourneau D, Wong R, Moseley D, Sharpe MB, Ansell S, Gospodarowicz $M$, Jaffray DA: Online planning and delivery technique for radiotherapy of spinal metastases using cone-beam CT: image quality and system performance. Int J Radiat Oncol Biol Phys 2007, 67:1229-37.

25. Siewerdsen JH, Jaffray DA: Optimization of $x$-ray imaging geometry (with specific application to flat-panel cone-beam computed tomography). Med Phys 2000, 27:1903-14.

26. Tucking T, Nill S, Oelfke U: Dose calculation on linac integrated KV-cone beam CT. Radiotherapy and oncology 2006, 81(Supplement 1):26.

27. Brock KK: Image registration in intensity-modulated, image-guided and stereotactic body radiation therapy. Front Radiat Ther Oncol 2007, 40:94-115.

28. Beaver ME, Matheny KE, Roberts DB, Myers JN: Predictors of weight loss during radiation therapy. Otolaryngol Head Neck Sur 2001, 125:645-8.

29. Hansen EK, Bucci MK, Quivey JM, Weinberg V, Xia P: Repeat CT imaging and replanning during the course of IMRT for head-and-neck cancer. Int J Radiat Oncol Biol Phys 2006, 64:355-62.

doi: $10.1186 / 1748-717 X-5-29$

Cite this article as: Hu et al., Use of kilovoltage X-ray volume imaging in patient dose calculation for head-and-neck and partial brain radiation therapy Radiation Oncology 2010, 5:29

Submit your next manuscript to BioMed Central and take full advantage of:

- Convenient online submission

- Thorough peer review

- No space constraints or color figure charges

- Immediate publication on acceptance

- Inclusion in PubMed, CAS, Scopus and Google Scholar

- Research which is freely available for redistribution

Submit your manuscript at www.biomedcentral.com/submit
Ciomed Central 